\title{
Broadcast Cancellation in Search Mechanisms
}

\author{
Rui Lima \\ Univ. Lusíada \\ CLEGI, V. N. Famalicão, PT \\ rml[at]fam.ulusiada.pt
}

\author{
Carlos Baquero \\ Univ. do Minho \& INESC Tec \\ HASLab, Braga, PT \\ cbm[at]di.uminho.pt
}

\author{
Hugo Miranda \\ Univ. de Lisboa \\ LaSIGE, Lisboa, PT \\ hmiranda[at]di.fc.ul.pt
}

\begin{abstract}
Searching for resources over unstructured networks is usually supported by broadcast communication primitives. Ideally, the broadcast process should be cancelled as soon as possible after a successful discovery, to avoid flooding the entire network. However, cancelling an ongoing broadcast is challenging and may increase the number of exchanged messages. In this paper, we compare the cancellation mechanisms used by BERS and BERS ${ }^{\star}$ with new proposed cancellation approaches BCIR and BCIR ${ }^{\star}$. The formulation of a simplified analytical model and the simulation results show that: i) it is possible to reduce the number of retransmitted messages, without increasing the latency observed in $\mathrm{BERS}^{\star}$; and ii) BCIR is more energy efficient, which can contribute to extend the availability of mobile battery powered devices.
\end{abstract}

\section{Categories and Subject Descriptors}

Networks [Network protocols]: Cross-layer protocols; Network types [Ad hoc networks]: Mobile ad hoc networks

\section{General Terms}

Protocols, Broadcast.

\section{Keywords}

Unstructured Networks, Mobile Systems, Resource Searching, Broadcast, Cancellation.

\section{INTRODUCTION}

\footnotetext{
* The work reported in this paper was co-financed by FCT - Fundação para a Ciência e Tecnologia, Portugal (PEst$\mathrm{OE} / \mathrm{EME} / \mathrm{UI} 4005 / 2011)$ and carried out within the research centre Centro Lusíada de Investigação e Desenvolvimento em Engenharia e Gestão Industrial (CLEGI).

${ }^{\dagger}$ Work described in this paper was partially supported by Fundação para a Ciência e Tecnologia (FCT) through project PTDC/EIA-EIA/103751/2008 - PATI.
}

Permission to make digital or hard copies of all or part of this work for personal or classroom use is granted without fee provided that copies are not made or distributed for profit or commercial advantage and that copies bear this notice and the full citation on the first page. To copy otherwise, to republish, to post on servers or to redistribute to lists, requires prior specific permission and/or a fee.

SAC'13 March 18-22, 2013, Coimbra, Portugal.

Copyright 2013 ACM 978-1-4503-1656-9/13/03 ...\$10.00.
In mobile multi-hop networks the node transmission range is smaller when compared with the network size, preventing direct radio communications between distant nodes. Some network nodes have to act as routers and relay the received messages, to maintain the network connectivity [1]. Also, the network topology changes over time, affected by the node mobility and by the radio transmission range. Any collected topology information is quickly outdated, making the classic routing principles useless. Typically unstructured wireless networks use broadcasting for data dissemination.

Broadcast [2] is a core building block for lower level services development, widely used by MANETs (Mobile Ad-hoc Networks) applications and protocols [8]. One application example is the search for resources (e.g. a specific node, a record of a given user's reputation or the device closest to some location), that can be found distributed over the network [11].

The network node limitations (energy, memory, communication, etc) promote the development of search mechanisms that improve critical resource usage by limiting non productive query message diffusion [4]. E.g. when searching for a given item or node, there is no utility in continuing the query dissemination, once the resource that matches the query is found. For sake of motivation, consider applications for emergency response scenarios that track response team positions by way of a multi-hop sensor network across several miles. In such setting, a query that searches a given team position should be interrupted once the team is found, instead of flooding the query through all device nodes in the network.

Some search mechanisms that are concerned with energy efficiency cancel an ongoing broadcast with the diffusion of stop messages in a subsequent limited broadcast $[3,6]$. This is achieved at the expense of adding fine tuned delays at each re-transmission step of the initial query propagation. Delays open a time window that gives the opportunity to block the original broadcast [12].

In contrast with previous approaches, that initiate the cancellation in the source node for the ongoing query, this paper proposes a new searching approach that starts the cancellation process at the node (nodes) where the resource is found, avoiding a communication step between the resource node and the initiator node. This approach results in two new searching mechanisms that explore the trade-off between latency and energy consumption.

This paper is structured as follows. Section 2 addresses related work on mechanisms that stop ongoing broadcasts. Then, Section 3 presents a new approach for broadcast can- 
cellation and includes a simplified analytical model used for predicting the cancellation mechanism behaviour. An evaluation and the simulation results are discussed in Section 4. Finally, Section 5 contains the conclusions and future research directions.

\section{RELATED WORK}

For unstructured wireless networks, the main problem addressed by searching mechanisms is to find if some node $n$ is holding a copy of some resource $r$ requested by some query initiator node $n_{0}$. We are considering that query matching answers are routed towards $n_{0}$ once instances of $r$ are found, and that at least one instance is available in the network. The query search is complete when node $n_{0}$ receives the first match, and thus can report it to a higher layer or an end user.

We now present a general description of a cancellation mechanism. Consider a multi-hop wireless network where the search process is initiated by node $n_{0}$, and $n_{H}$ is the node (or one of the nodes) that has the nearest resource copy, and is located at $H$ hops from $n_{0}$. The search process is initiated in node $n_{0}$ with the transmission of a search/query message $m_{s}$, to be disseminated using the intermediate nodes to forward (relay) the packets. When $n_{H}$ receives the message $m_{s}$, a point-to-point (following the reverse route that is piggybacked to $m_{s}$ transmissions) answer message $m_{a}$ is sent from $n_{H}$ to $n_{0}$, to notify the discovery. The search process assumes that any node at any instant can start the diffusion of a cancellation message $m_{c}$ to stop the propagation of $m_{s}$.

System Model. We consider a system model, in line with the related work, where nodes transmit in synchronized rounds and form network topologies that follow a random geometric distribution, i.e. nodes are random uniformly spread in a plane and two nodes are connected if their distance is under a given threshold. We are interested in finding resources that are in the connected component of the querying node, and, without loss of generality, consider that the network has a single connected component. In this analysis we do not consider failures in neither nodes nor transmissions.

Cancellation at the same hop. One of the mechanisms proposed for better energy efficient cancellation is the Blocking Expanding Ring Search (BERS) [3]. BERS is depicted in Fig. 1. During the forward process, the search message $m_{s}$ is forwarded under a parametric delay, adding a waiting time that is double of the hop count delay $=2 \times$ hop, measuring each node's hop distance from $n_{0}$. When $m_{s}$ reaches $n_{H}$ and the resource is discovered, $n_{H}$ sends the point-to-point answer message $m_{a}$ back to the initiator $n_{0}$. The message $m_{a}$ is relayed without any additional delays, and through the shortest path. When the initiator $n_{0}$ receives $m_{a}$, it starts the cancellation process by broadcasting the cancellation message $m_{c}$. The message $m_{c}$ is relayed with no additional delays by all the intermediate nodes that relayed the message $m_{s}$. Considering $t_{a}$ as the time (number of synchronous transmission rounds) spent by $m_{a}$ to be relayed from $n_{H}$ to $n_{0}$ and $t_{c}$ as the time spent by $m_{c}$ to be propagated until hop $H$. Assuming that messages $m_{a}$ and $m_{c}$ have the same size and can be transmitted in a single round, both messages will take the same time to reach to destination, namely: $t_{a}=t_{c}=H$. The added delay $2 \times H$ (again in terms of transmission rounds, that are to be waited) imposed in BERS for $m_{s}$ enables the time windows to allow the cancellation at the same hop where the resource

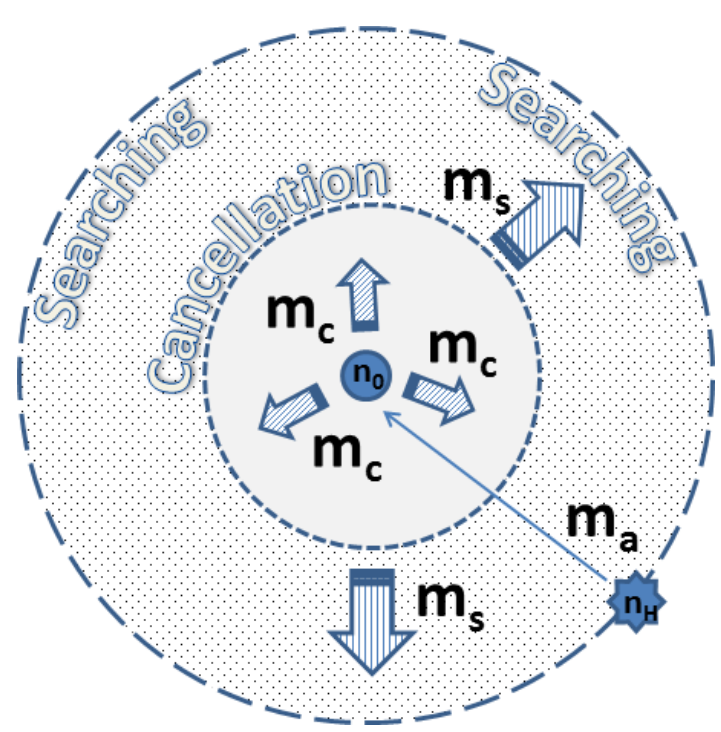

Figure 1: BERS - Blocking Expanding Ring Search

is found. The nodes belonging to hop $H$ do not relay any message, because they receive $m_{s}$ and $m_{c}$ during the same slot time and the broadcast process stops.

Cancellation at the next hop. Naturally, adding a delay increases the latency observed from query initiation until the first answer is delivered. One strategy to decrease the latency observed in BERS is to reduce the delay added during $m_{s}$ retransmissions. The enhanced BERS (BERS $\left.S^{\star}\right)$ mechanism is similar to BERS and the only difference is the reduction to half of the delay time of BERS, making delay $=H$ [6]. Lowering the added delay has consequences in energy efficiency, because there is no time to propagate both messages $m_{a}$ and $m_{c}$ before the nodes in hop $H$ start relaying for next hop. The resulting behaviour is the continuation of $m_{s}$ diffusion for one additional hop. The BERS* cancellation mechanism stops one hop after the discovery, because only during the slot time $H+1$ both $m_{s}$ and $m_{c}$ are received by the nodes at $H+1$ hops, and the search process is finished.

Discussion. Considering only searching mechanisms where the broadcast primitive is contained by adding delays to the retransmission of the query messages, it is observed that BERS is more energy efficient than BERS ${ }^{\star}$, because BERS ${ }^{\star}$ generates more retransmissions by the nodes in the additional hop $H+1$. However, BERS ${ }^{\star}$ has less latency than BERS, because the added delay is reduced. The trade-off between the energy consumption and latency is a fundamental characteristic of the expanding ring searching mechanisms, that can be explored by new approaches [5].

\section{BROADCAST CANCELLATION INITIATED ON RESOURCE (BCIR)}

In contrast with BERS and BERS ${ }^{\star}$, we propose a new mechanism BCIR (Broadcast Cancellation Initiated on Resource), where the cancellation process is initiated by the node $n_{H}$, avoiding the waiting time taken for $m_{a}$ propagation. BCIR has an immediate impact in the neighbourhood of $n_{H}$. As depicted by Fig. 2, the diffusion of message $m_{c}$ starts at the searching ring periphery, and no 


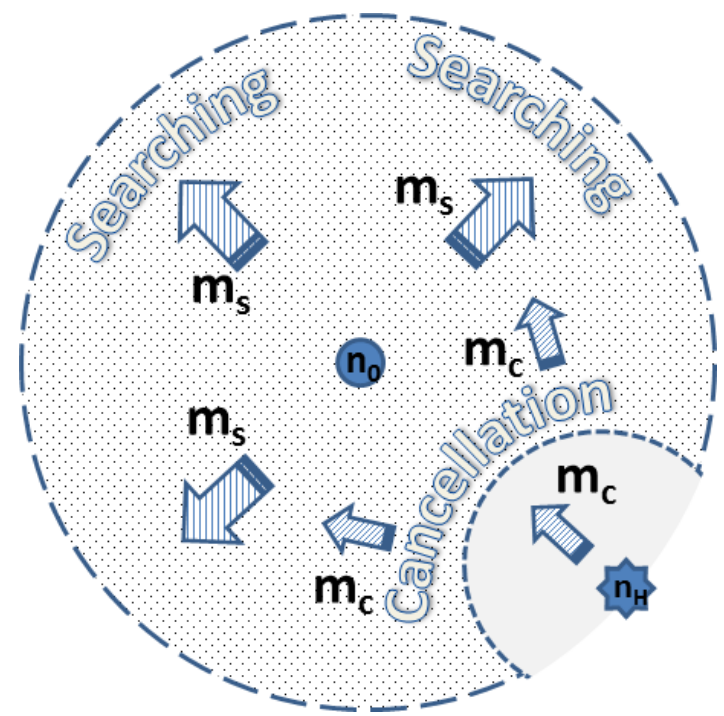

Figure 2: BCIR - Broadcast Cancellation Initiated on Resource

\begin{tabular}{l|rrrrrr}
$H$ & 1 & 2 & 3 & 4 & 5 & $\ldots$ \\
\hline $\mathrm{t}(\mathrm{H})$ & 1 & 4 & 9 & 16 & 25 & $\ldots$
\end{tabular}

Table 1: $m_{s}$ time sequence for BERS and BCIR

delay is added to the diffusion of $m_{c}$. If multiple copies are discovered on the same hop $(H)$, multiple cancellation messages diffusions will be initiated, thus decreasing the time required to cancel the entire broadcast.

Similar to BERS and BERS ${ }^{\star}$ the added delay for BCIR generates two variants. The BCIR with a $2 \times H$ delay and the BCIR $^{\star}$ with a $H$ delay. These variants allows to study the added delay impact in energy efficiency and latency. To compare the new cancellation mechanisms BCIR and BCIR ${ }^{\star}$ with the BERS and BERS ${ }^{\star}$, we proceeded for an analytical modelling using two distinct metrics: latency and retransmission rates. The analytical modelling enables us to check how the cancellation initiated from $n_{0}$ relates with the cancellation initiated from $n_{H}$.

Latency $(\mathbf{L})$. The latency $L_{H}$ can be defined as the time between the start of the broadcasting searching message $m_{s}$ by node $n_{0}$ and the time when the node $n_{0}$ receives a successful answer to the initial query. We are assuming a multihop network scenario where latency can be expressed as a function of $H$, given that radio signal propagation delay is negligible compared to the intentionally added delay introduced by the nodes.

In both mechanisms BERS and BCIR, any relay node $n_{i}$ at $i$ hops from $n_{0}$ will delay the retransmission by $2 \times i$, resulting in a time progressing function given by Eq. 1 recursive expression:

$$
t(H)= \begin{cases}1, & H=1 \\ t_{(H-1)}+2 \times(H-1)+1, & H \geq 2\end{cases}
$$

The Eq. 1 results in the time sequence of Tab. 1. Applying polynomial interpolation to the sequence of Tab. 1, we obtain the expression for the time instant when $m_{s}$ reaches a

\begin{tabular}{l|rrrrrr}
$H$ & 1 & 2 & 3 & 4 & 5 & $\ldots$ \\
\hline$t(H)$ & 1 & 3 & 6 & 10 & 15 & $\ldots$
\end{tabular}

Table 2: $m_{s}$ time sequence for $\mathrm{BERS}^{\star}$ e $\mathrm{BCIR}^{\star}$

node at hop $H$ :

$$
t(H)=H^{2}
$$

The latency $(L)$ of BERS and BCIR mechanisms will be the same. Assuming that $m_{a}$ is propagated as quickly as possible (no added delay), i.e. the delay is given by $t_{a}=H$, and combining with Eq. 2, results the latency expression in Eq. 3

$$
L_{B E R S}=t_{s}+t_{a}=H^{2}+H=L_{B C I R}
$$

Knowing that in $\mathrm{BERS}^{\star}$ and $\mathrm{BCIR}^{\star}$ mechanisms, a node $n_{i}$ delays the retransmission by $i, t(H)$ is given by Eq. 4 recursive expression:

$$
t(H)= \begin{cases}1, & H=1 \\ t_{(H-1)}+(H-1)+1, & H \geq 2\end{cases}
$$

The expression in Eq. 4 results in the time sequence of Tab. 2. Applying polynomial interpolation to the sequence in Tab. 2, we obtain the expression for the time instant when $m_{s}$ reaches a node at hop $H$ :

$$
t(H)=\frac{H^{2}+H}{2}
$$

For the latency of $\mathrm{BERS}^{\star}$ and $\mathrm{BCIR}^{\star}$, it is necessary to add $t_{a}$, thus resulting in the expression on Eq. 5:

$$
L_{B E R S^{\star}}=t_{s}+t_{a}=\frac{H^{2}+3 H}{2}=L_{B C I R^{\star}}
$$

Fig. 3 compares the latency for several cancellation mechanisms with respect to the distance between the resource and $n_{0}$. The graph latency starts for $H=1$, since $H=0$ is the case when $n_{0}$ is holding the resource. As $H$ increases, more nodes will be contacted to find the resource, i.e. the resources are further away from $n_{0}$. The BERS ${ }^{\star}$ and BCIR ${ }^{\star}$ mechanisms improve their latency gains when the number of hops to find the resource increases, and therefore will have better performance for larger networks with a low-density of resources.

Retransmissions ratio $(\mathbf{R})$. The retransmission ratio (R) corresponds to the ratio between the number of retransmissions required until the searching process ends and the number of network nodes. The retransmission ratio $(\mathrm{R})$ calculation is independent of the delivery of $m_{a}$. It considers all the retransmissions until the broadcast is completely cancelled. Assuming that the energy spent in each node is evenly distributed across all network nodes $(N)$ and that each retransmission consumes the same amount of energy, then the total energy consumed is roughly proportional to the retransmission rate.

Considering the node density to be $\sigma$, i.e. the number of nodes per unit area, we can assume that for a constant node density and omnidirectional propagation, the query dissemination until the hop $H$ is relayed by $N_{H}$ nodes, according to Eq. 7:

$$
N_{H}=\sigma \times \pi \times H^{2}
$$




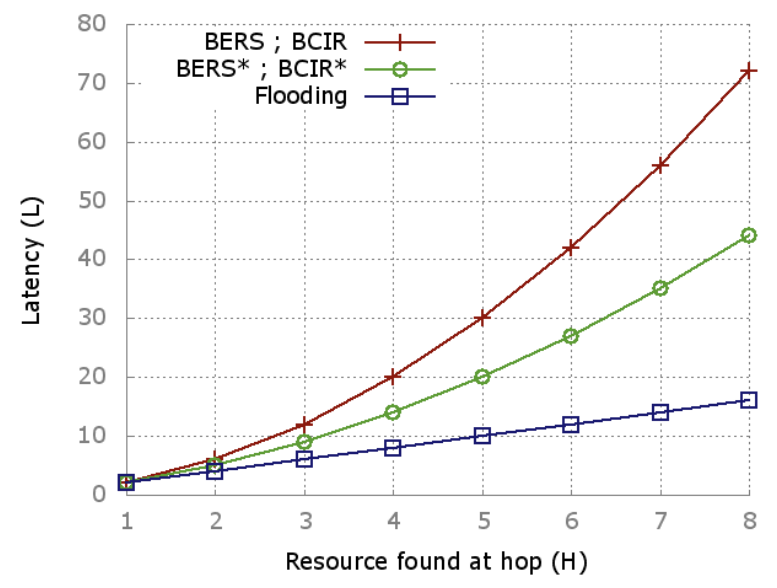

Figure 3: Analytical Results: Latency (T)

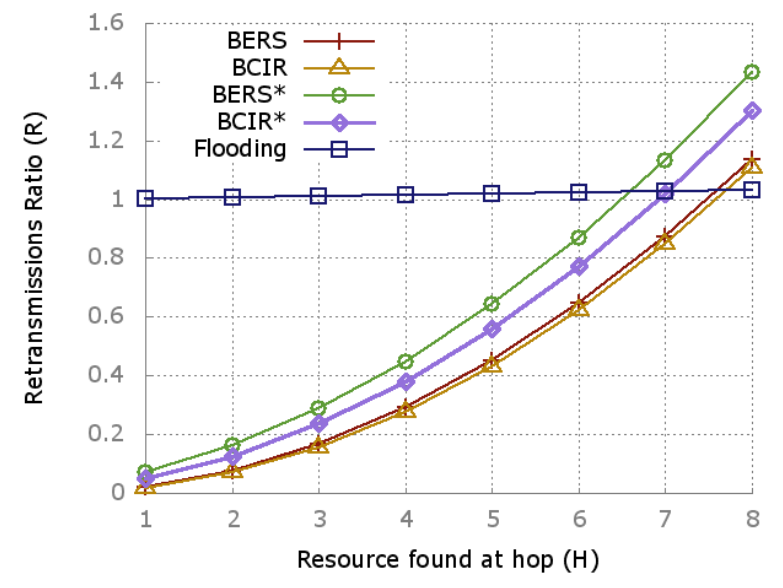

Figure 4: Analytical Results: Normalized retransmissions ratio $(R)$

If the number of retransmissions is proportional to $H^{2}$, then the expression for the retransmission ratio related with the message dissemination process done by the nodes within the ring $H$ is:

$$
R_{H}=\frac{N_{H}}{N}=\frac{\sigma \pi H^{2}}{N}
$$

The retransmissions ratio $(\mathrm{R})$ for BERS, BERS* and BCIR is given by the following expressions, where $r_{s}$ are the retransmissions ratio for the searching dissemination, and $r_{r}$ are the retransmissions ratio for point-to-point answer message, and $r_{c}$ are the retransmission rates for the cancellation process.

$$
\begin{gathered}
R_{B E R S}=r_{s}+r_{r}+r_{c}=\frac{\sigma \pi H^{2}}{N}+\frac{H}{N}+\frac{\sigma \pi H^{2}}{N}=\frac{2 \sigma \pi}{N} H^{2}+\frac{1}{N} H \\
R_{B E R S^{\star}}=r_{s}+r_{r}+r_{c}=\frac{2 \sigma \pi}{N}(H+1)^{2}+\frac{1}{N} H \\
R_{B C I R}=r_{s}+r_{c}=\frac{\sigma \pi H^{2}}{N}+\frac{\sigma \pi H^{2}}{N}=\frac{2 \sigma \pi}{N} H^{2}
\end{gathered}
$$

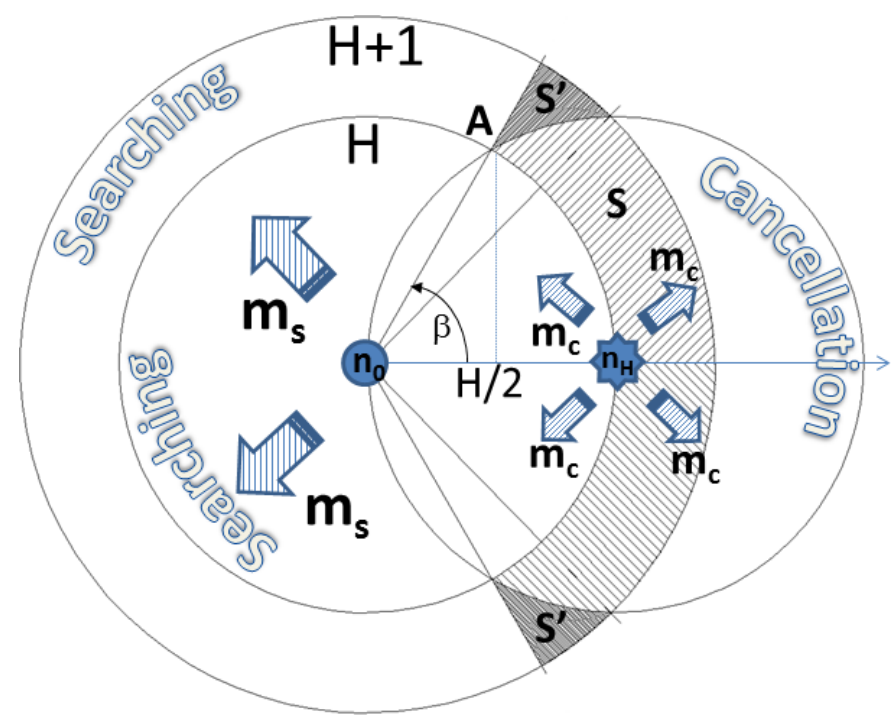

Figure 5: BCIR $^{\star}$ cancellation mechanism

Fig. 5 shows how the BCIR $^{\star}$ cancellation mechanism affects the $n_{H}$ neighbour nodes. Nodes receiving a message $m_{c}$ before the message $m_{s}$ retransmission moment, will not relay both messages $m_{s}$ and $m_{c}$. This early cancellation effect cannot be neglected, since some of the nodes belonging to the ring $H+1$ will not retransmit the message $m_{s}$ and the retransmissions are automatically cancelled.

The $\mathrm{BCIR}^{\star}$ retransmissions ratio (R) must consider the nodes belonging to region $S$ depicted in Fig. 5, that will not relay both messages $m_{s}$ and $m_{c}$, because they already received at least one cancellation message. The region $S$ corresponds to an energy gain.

To simplify the analytical expression, instead using the area region $S$ for calculation, we consider the area region $S_{T}=2 S^{\prime}+S$. The $S_{T}$ area is depicted in Fig. 5 and can be expressed in the cylindrical coordinates system, according to Eq. 12 , where point $A$ has coordinates $\left(\frac{H}{2}, \frac{\sqrt{3} H}{2}\right)$ and an angle $\beta=\frac{\pi}{3}$.

$$
S_{T}=\int_{H}^{H+1} \int_{-\pi / 3}^{\pi / 3} r d r d \phi=\frac{\pi}{3}(2 H+1)
$$

The Eq. 12 reveals a linear dependence between the cancellation area $\left(S_{T}\right)$ and the distance $(H)$. The number of nodes corresponding to the cancellation area can be obtained by the expression:

$$
N_{S_{T}}=\frac{1}{3}\left(N_{H+1}-N_{H}\right)=\frac{\sigma \pi}{3}(2 H+1) \simeq N_{S}
$$

which is according to the Eq. 12

For the $\mathrm{BCIR}^{\star}$ cancellation mechanism, the retransmission rates are obtained using Eq. 11, but now considering $H+1$ hops and knowing that $N_{S}$ will not relay the $m_{s}$ and $m_{c}$ messages. Therefore it is necessary to remove these $2 N_{S}$ retransmissions, i.e.:

$$
R_{B C I R^{\star}} \simeq R_{B C I R_{H+1}}-2\left(\frac{N_{S}}{N}\right)
$$


Using Eq. 14 and Eq. 12, results:

$$
R_{B C I R^{\star}} \simeq \frac{2 \sigma \pi}{3 N}\left(3 H^{2}+4 H+2\right)
$$

Combining the Eq. 10 and 15 results the expression for the energy efficiency gain $\Delta_{R}$ of $\mathrm{BCIR}^{\star}$ over the $\mathrm{BERS}^{\star}$, that can be quantified as the difference between the respective retransmissions ratio, i.e.:

$$
\Delta_{R}=R_{B E R S^{\star}}-R_{B C I R^{\star}} \lesssim \frac{2 \sigma \pi}{3 N}(2 H+1)+\frac{1}{N} H
$$

By fixing $N$ and $\sigma$, we can calculate the retransmission ratio using equations $9,10,11$ and 15 . Fig. 4 compares the retransmissions ratio for BERS, BERS ${ }^{\star}$, BCIR and $\mathrm{BCIR}^{\star}$ cancellation mechanisms.

When interpreting the graph in Fig. 4 is interesting to note that the $\mathrm{BCIR}^{\star}$ retransmission ratio are below the $\mathrm{BERS}^{\star}$, showing that $\mathrm{BCIR}^{\star}$ will save energy over BERS ${ }^{\star}$, but maintaining the same latency (Fig. 3) of BERS ${ }^{\star}$.

The size and the network topology will impose the $H_{\max }$ i.e. the maximum number of hops or the network diameter. Any flooding broadcast will stop by itself, with an energy cost proportional to $H_{\max }^{2}$. The cancellation mechanisms wastes less energy than a simple flooding, when the resource is found bellow the $H \leq \frac{H_{\max }}{\sqrt{2}}$ hops threshold. Otherwise, flooding is the better solution as depicted in Fig. 4.

\section{EVALUATION}

Simulations were used for the comparison of the search mechanisms under the metrics defined in Sec 3 (Latency and Retransmissions ratio), and uses round based simulation. Although the simulation engine enables us to do some experimentations in different scenarios and topologies, the focus here will cover random geometric networks. We note, however, that cancellation can also be applied to wide area overlay networks over the Internet, and other topologies are relevant for that setting.

Simulation Environment. We developed a high-level simulation environment using random geometric topologies, that simplifies the MANET communication model [7]. For each searching operation the simulator generates a new random network topology and randomly spread resources to the network nodes. Assuming a sufficiently small duration for each diffusion, it is conceivable that the network topology remains the same during the searching process. The random geometric topologies match most of the BCIR application scenarios for WUSN (Wireless Underground Sensor Networks) $[10,9]$. The radio transmission range is modelled by the graph $G$ connectivity where each node has its limited range. Each graph $G=(V, E)$ is defined by a set of vertices $V$ (which represent the network nodes) and a set of links between the vertices $E$ (representing the radio range between each node pair).

Results. The average results depicted in figures 6, 7(a) and 7(b), are obtained after 2000 simulation runs, for each resource density. To improve graph readability in Fig. 6 resource densities exceeding $25 \%$ are not represented, since the probability of finding the resource in the initiator $n_{0}$ neighbourhood is high and all curves will overlap.

In general, the analytical model predictions are confirmed by the simulation results. If $H_{\max }$ is known, the analytical model establish a threshold of $\frac{H_{\max }}{\sqrt{2}}$ for choosing be-

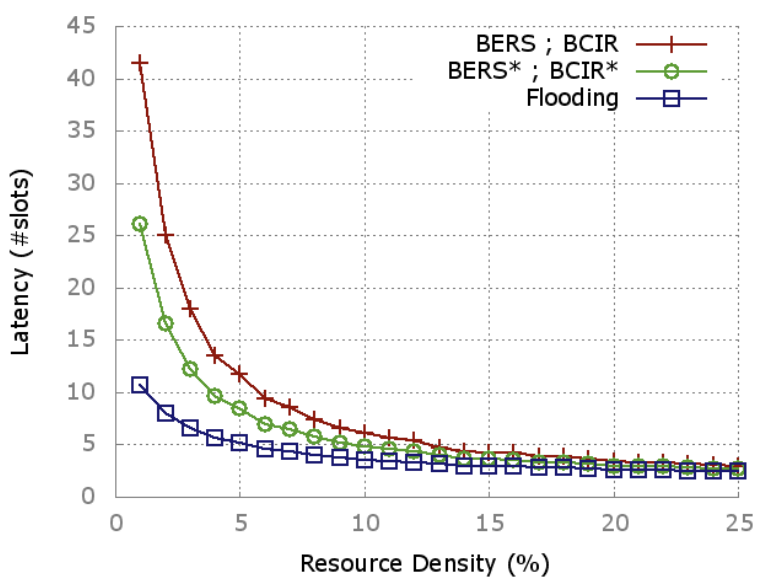

Figure 6: Simulation Results - Latency (T)

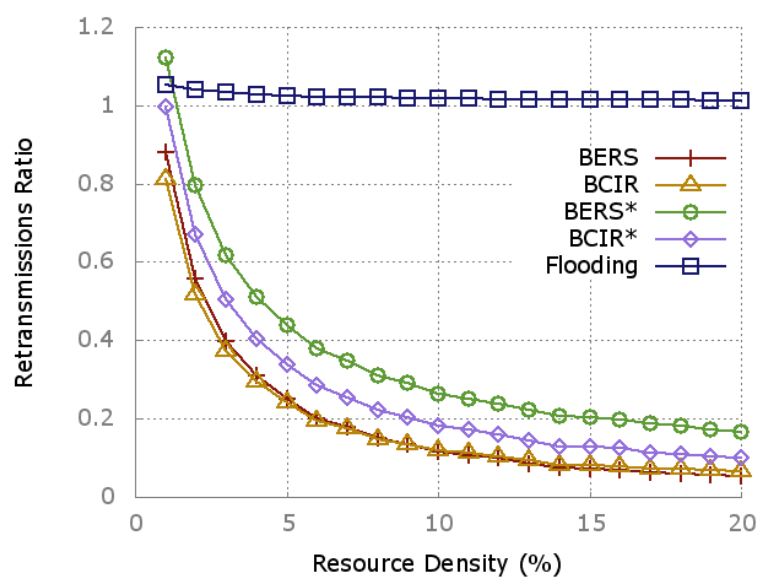

(a) Low Resources

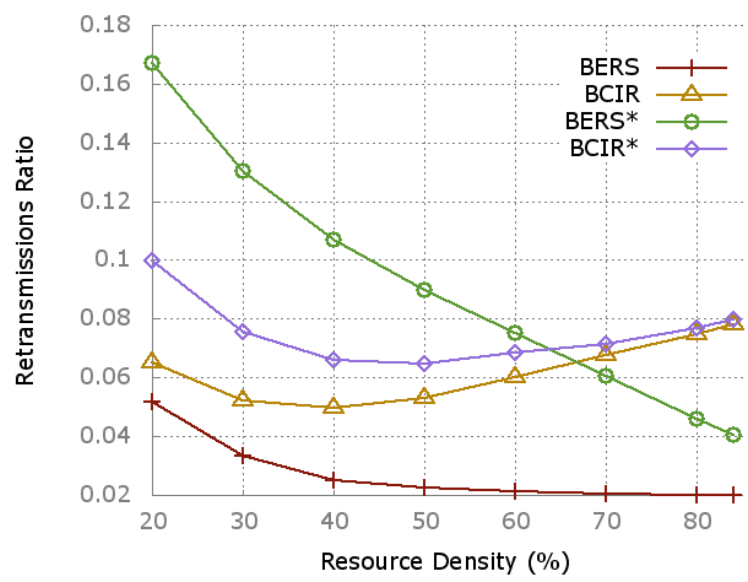

(b) High Resources

Figure 7: Simulation Results - Retransmissions ratio (R)

tween applying the cancellation mechanisms or doing nothing, since then flooding becomes the best solution.

As expected, BERS ${ }^{\star}$ and $\mathrm{BCIR}^{\star}$ exhibit significantly lower 
latencies than BERS and BCIR, as a result from the smaller delay during the search phase. The graph analysis depicted in Fig. 6, allow us to observe that both BCIR ${ }^{\star}$ and BERS ${ }^{\star}$ have the same latency, and the same happens for their longer delay variants. The flooding retransmission ratio for resource densities over $20 \%$ converges to 1 . This is why flooding is not present at Fig. 7(b).

The main result is that $\mathrm{BCIR}^{\star}$ while keeping a low latency impact, can still perform better that BERS ${ }^{\star}$ across most density ranges thus enabling important energy savings, as depicted in Fig. 7(b).

Equation 16 alerts that if $\mathrm{BERS}^{\star}$ is replaced by $\mathrm{BCIR}^{\star}$ then the energy saving $\Delta_{R}$ increases linearly with $H$. For a given node density $\sigma$, when more hops are required for a successfully resource discovery, more justified is the replacement by the new mechanism BCIR ${ }^{\star}$.

The worst performance for retransmission ratios under resource percentages in excess of $60 \%$ was a result that was not anticipated by the analytical model. This deviation is a consequence of the resource density increment, and the corresponding probability increase of finding multiple resources on the same hop $(H)$, which in turn will lead to multiple concurrent cancellation waves (in contrast with a single cancellation wave from the source node). The diffusion of multiple cancellation waves may result (depending on the network topology) in an overlap frontier where BCIR can introduce a duplication of cancellation messages. The nodes have no information on the status of its neighbours and the multi-cancellation broadcasts will slight increase the retransmissions ratio for nodes belonging to distinct cancellation waves.

The $\mathrm{BCIR}^{\star}$ mechanism proved to be very efficient for networks with low resources density. As depicted at Fig. 7, the increase in energy gain has more impact for networks with resource densities less than $40 \%$. The BCIR ${ }^{\star}$ characteristics, give it the potential to be more efficient in large networks, assuming that the resource density decreases when the network size is bigger, as highlighted by the motivating emergency response team example. Confirming these results in networks with different node compositions and structure is relegated to future work.

\section{CONCLUSIONS}

This paper compares the broadcasting cancellation search mechanism BERS and BERS ${ }^{\star}$ with two variants of a novel algorithm named BCIR and BCIR ${ }^{\star}$. The cancellation mechanism analysis led to the establishment of an analytical modelling, which allowed to find expressions for latency and retransmission rates, and contrast them with simulation results.

The main conclusion is that the mechanism BCIR ${ }^{\star}$ proved to be more efficient than $\mathrm{BERS}^{\star}$, with respect to the retransmissions ratio. Our proposed algorithm for BCIR, where the cancellation is immediately initiated by the nodes where the resource is found, has proved to be more suitable for MANETs networks. Results show that it is possible to obtain a latency at least equal to the faster competing mechanism, with a lower energy cost.

In the future we intend to evaluate BCIR taking in account the radio propagation models and node mobility (e.g. under ns2). Future simulations should explore the impact of a reduction in the added delay, progressing to values below the value used in $\mathrm{BCIR}^{\star}$, to improve latency and measuring the consequences in energy expenditure.

\section{REFERENCES}

[1] A. Boukerche, B. Turgut, N. Aydin, M. Z. Ahmad, L. Bölöni, and D. Turgut. Routing protocols in ad hoc networks: A survey. Computer Networks, 55(13):3032-3080, Sept. 2011.

[2] Z. Cheng and W. B. Heinzelman. Flooding strategy for target discovery in wireless networks. Wirel. Netw., 11:607-618, September 2005.

[3] I. Park, J. Kim, and I. Pu. Blocking expanding ring search algorithm for efficient energy consumption in mobile ad hoc networks. In WONS 2006 : Third Annual Conference on Wireless On-demand Network Systems and Services, pages 191-195, Les Ménuires (France), Jan. 2006. INRIA, INSA Lyon, IFIP, Alcatel. http://citi.insa-lyon.fr/wons2006/index.html.

[4] I. Park and I. Pu. Energy efficient expanding ring search. In Modelling Simulation, 200\%. AMS '0\%. First Asia International Conference on, pages 198 -199, march 2007.

[5] I. Pu, J. Kim, and Y. Shen. Energy-time efficiency of two routing strategies with chase packets in expanding ring search. In Telecommunications (ICT), 2010 IEEE 17th International Conference on, pages $742-747$, april 2010.

[6] I. Pu and Y. Shen. Enhanced blocking expanding ring search in mobile ad hoc networks. In New Technologies, Mobility and Security (NTMS), 2009 3rd International Conference on, pages $1-5$, dec. 2009.

[7] Y. Shang. Exponential random geometric graph process models for mobile wireless networks. In Cyber-Enabled Distributed Computing and Knowledge Discovery, 2009. CyberC '09. International Conference on, pages $56-61$, oct. 2009.

[8] S. Suman and Balkrishan. Enhanced aodv for wireless networks. In Advance Computing Conference (IACC), 2010 IEEE 2nd International, pages 246 -249, feb. 2010.

[9] Z. Sun, I. Akyildiz, and G. Hancke. Dynamic connectivity in wireless underground sensor networks. Wireless Communications, IEEE Transactions on, PP(99):1 -11, 2011.

[10] C. Wenqi and X. Zhao. Multi-hop routing for wireless network in underground mines. In Wearable Computing Systems (APWCS), 2010 Asia-Pacific Conference on, pages $337-340$, april 2010.

[11] M. B. Yassein, S. F. Nimer, and A. Y. Al-Dubai. A new dynamic counter-based broadcasting scheme for mobile ad hoc networks. Simulation Modelling Practice and Theory, 19(1):553 - 563, 2011. Modeling and Performance Analysis of Networking and Collaborative Systems.

[12] X. Zhang, T. Kunz, L. Li, and O. Yang. An energy-efficient broadcast protocol in manets: Design and evaluation. Communication Networks and Services Research, Annual Conference on, 0:199-206, 2010. 